\title{
Vibration-tolerant narrow-linewidth semiconductor disk laser using novel frequency-stabilisation schemes
}

\author{
Craig R. Hunter ${ }^{*}, 1,2$, Brynmor E. Jones ${ }^{1}$, Peter Schlosser ${ }^{1}$, Simon Toft Sørensen ${ }^{1}$, Michael J. Strain ${ }^{2}$, \\ Loyd J. McKnight ${ }^{1}$ \\ ${ }^{1}$ Fraunhofer Centre for Applied Photonics, 99 George Street, Glasgow, G1 1RD, UK \\ ${ }^{2}$ Institute of Photonics, University of Strathclyde, 99 George Street, Glasgow, G1 1RD, UK
}

\begin{abstract}
This paper will present developments in narrow-linewidth semiconductor-disk-laser systems using novel frequencystabilisation schemes for reduced sensitivity to mechanical vibrations, a critical requirement for mobile applications. Narrow-linewidth single-frequency lasers are required for a range of applications including metrology and highresolution spectroscopy. Stabilisation of the laser was achieved using a monolithic fibre-optic ring resonator with free spectral range of $181 \mathrm{MHz}$ and finesse of 52 to act as passive reference cavity for the laser. Such a cavity can operate over a broad wavelength range and is immune to a wide band of vibrational frequency noise due to its monolithic implementation. The frequency noise of the locked system has been measured and compared to typical Fabry-Perotlocked lasers using vibration equipment to simulate harsh environments, and analysed here. Locked linewidths of $<40 \mathrm{kHz}$ have been achieved. These developments offer a portable, narrow-linewidth laser system for harsh environments that can be flexibly designed for a range of applications.
\end{abstract}

Keywords: SDL, VECSEL, single-frequency, narrow-linewidth, stabilisation, ring-resonator, frequency-noise

\section{INTRODUCTION}

This paper presents developments in narrow-linewidth semiconductor-disk-laser (SDL) ${ }^{1,2}$ systems using novel frequencystabilisation schemes and compares them with conventional systems used for stabilisation, testing each system in harsh environments to emulate that which can be expected outside of a laboratory.

Narrow-linewidth single-frequency lasers are required for a range of applications including metrology, interferometry, Doppler LIDAR, atomic cooling and spectroscopy. Many of these applications require narrow-linewidth lasers at a range of wavelengths whether for optimised transmission/detection through different media or to address specific transitions in specific atoms, therefore, the wavelength flexibility due to bandgap engineering of SDL systems is an attractive solution for a range of applications.

SDLs can readily offer power levels beyond $1 \mathrm{~W}^{3}$ and it has been shown that more than $20 \mathrm{~W}^{4}$ is achievable for singlefrequency operation. Wavelength versatility is afforded by wide portfolio of available semiconductor materials ${ }^{2}$, with SDLs having been reported ranging from the $\mathrm{UV}^{5,6}$ to the mid-IR ${ }^{7,8}$. In order to further engineer these sources to provide the desired laser characteristics, intra-cavity elements can be deployed that allow for single-frequency operation, intracavity frequency conversion or modelocking. The SDL system offers a spectrally pure output without any background amplified spontaneous emission due to the lower gain and high quality cavity.

Reference cavities are essential components in order to narrow the linewidth of a laser system, an essential criterion for many applications. The prevalent reference cavity used currently is a Fabry-Perot interferometer ${ }^{7-9}$, which is typically a confocal cavity formed of distinct high reflector mirrors mounted on a spacer material. Consequently, this is sensitive to vibrations, leading to fluctuations in the length of the cavity and therefore fluctuations in the resonant frequency of the reference cavity. A monolithic reference cavity, such as the fibre-cavity demonstrated here will not suffer from misalignment or mirror separation problems caused by vibrations, and can be operated over the full transparency range of silica without the need for specialised optical coatings. Furthermore, single-frequency fibre lasers routinely offer

* craig.hunter.101@strath.ac.uk 
linewidths below $2 \mathrm{kHz}^{10}$ that benefit from the monolithic nature of the cavity. Therefore, the combination of the SDL and fibre loop offers the benefits of wavelength flexibility with frequency stability in a low-cost, compact format, ideal for mobile applications.

For these applications the measurement of linewidth is critical but remains challenging. There is no standardised method or timescale for calculating linewidth, and linewidths have been quoted over a range of different timeframes in literature ${ }^{5,8}$, and can be calculated using a range of different methods ${ }^{5,8,11}$. Here we focus on calculating the linewidth by measuring the frequency noise power spectral density (PSD) ${ }^{5,9}$ and provide a comparison to the self-heterodyne method $^{11}$.

\section{EXPERIMENTAL SETUP}

A compact, $808 \mathrm{~nm}$ diode-pumped SDL geometry operating at $1055 \mathrm{~nm}$ was developed on a $16 \mathrm{~cm} \times 7 \mathrm{~cm}$ baseplate. The gain chip was a resonant multiple quantum-well InGaAs/GaAs with AlGaAs/GaAs distributed Bragg reflector. The gain chip was secured to a temperature controlled mount, with an intra-cavity diamond heatspreader ${ }^{12}$ bonded to the front of the chip for power scaling. The diamond was plane-parallel and therefore also acts as an etalon in the cavity. In order to passively stabilise the free-running linewidth of the SDL, all water-cooling and cooling fans were removed from the system. A custom designed baseplate was fabricated for the laser cavity and temperature controlled using a thermoelectric cooler (TEC). The $126 \mathrm{~mm}$ laser cavity was completed by a $5 \%$ output coupler attached to a piezo actuator (PZT), and a folding mirror with a radius of curvature of $75 \mathrm{~mm}$. The cavity was optimised for single-transverse-mode operation and kept short in order to assist with single-frequency operation.

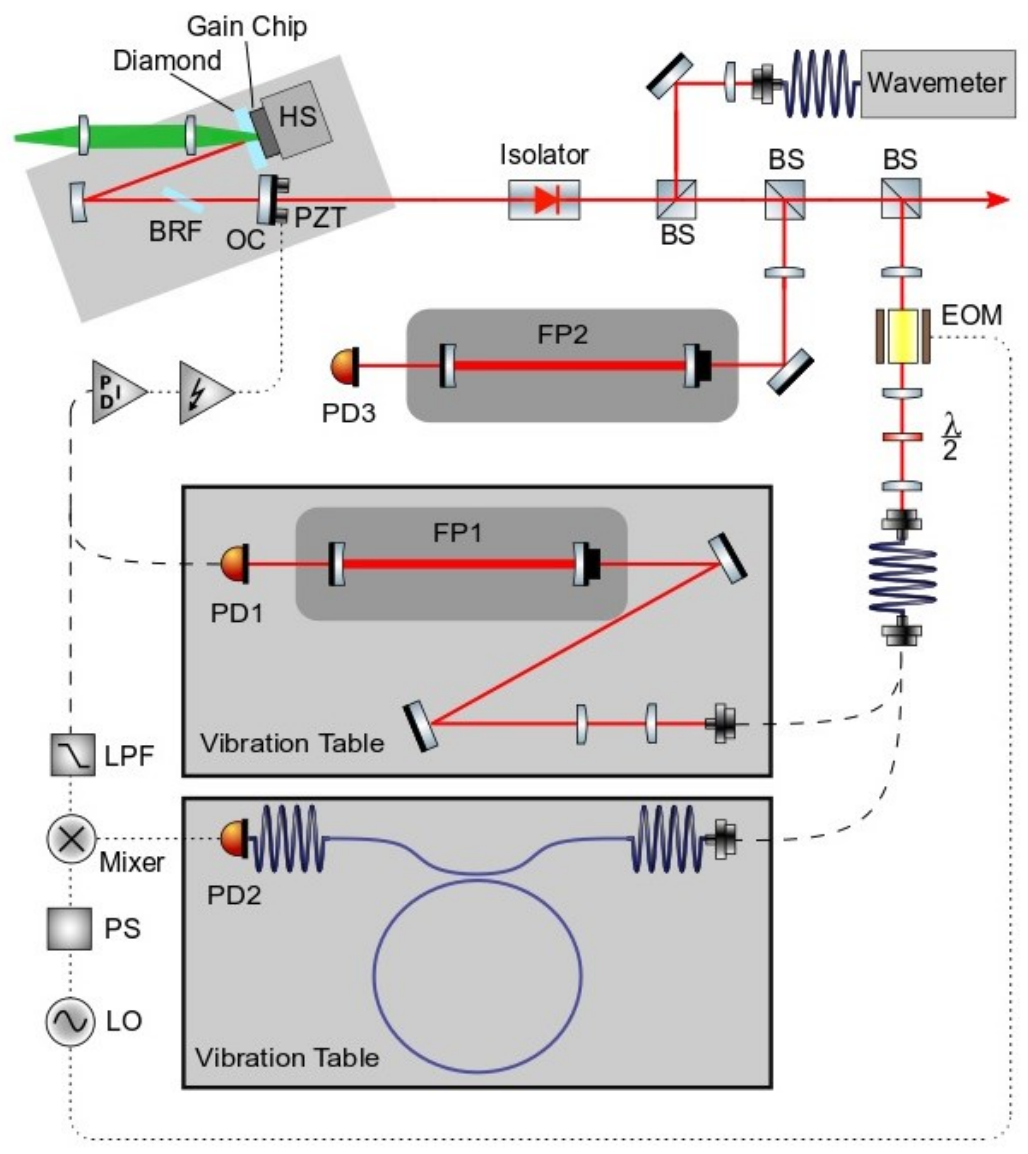

Figure 1. Schematic of set-up showing two paths for locking to the different reference cavities. OC $=$ output coupler, $\mathrm{PZT}=$ piezo actuator, $\mathrm{BRF}=$ birefringent filter, $\mathrm{HS}=$ heatsink, $\mathrm{BS}=$ beamsplitter, $\mathrm{EOM}=$ electro-optic modulator, $\lambda / 2=$ half-wave plate, $\mathrm{FP}=$ Fabry-Perot Interferometer, $\mathrm{PD}=$ Photodiode, $\mathrm{LO}=$ local oscillator, $\mathrm{PS}=$ phase shifter, $\mathrm{LPF}=$ low pass filter 
Active stabilisation of the laser was achieved either by locking it to a Fabry-Perot reference cavity using the side-of-fringe locking technique ${ }^{8,9}$, or by locking it to a fibre-cavity using the Pound-Drever-Hall (PDH) locking technique ${ }^{13,14}$. The sideof-fringe technique suffers from being unable to discern between fluctuations in the frequency of the laser, and fluctuations in the intensity of the laser itself, which will have a direct effect on the linewidth. The PDH locking technique is insensitive to fluctuations in the intensity of the laser.

The fibre-cavity was assembled using low-cost commercially available components and use of a Fujikura FSM-100P+ fibre-splicer. The connections of a $2 \times 2$ polarisation-maintaining fibre coupler were spliced together to make a ring resonator. A 99:1 coupler was used to obtain a resonance suitable for locking and polarisation-maintaining fibre was used to prevent polarised mode-splitting caused by birefringence in stressed fibre. The fibre was stripped of its outer jacket to minimise internal vibrations and then secured to an aluminium baseplate using UV-cured epoxy to reduce stresses on the fibre-cavity.

\section{FREE RUNNING LASER OPERATION}

Single-frequency operation at $1055 \mathrm{~nm}$ was obtained by using an intracavity $4 \mathrm{~mm}$ thick quartz birefringent filter (BRF), as shown in Figure 2a, with output powers up to $1.8 \mathrm{~W}$, a slope efficiency of $29.5 \%$, and a $\mathrm{M}^{2}$ of $<1.1$. A coarse-tuning range of $20 \mathrm{~nm}$ was achieved by rotation of the BRF, with a mode-hop-free fine-tuning range of $1 \mathrm{GHz}$ facilitated by the PZT. Single-frequency operation was obtained without any additional etalon owing to the short cavity length, high output coupling and filtering effects of the intracavity diamond and gain chip. The wavelength was monitored using a HighFinesse WS7 wavemeter over a period of 30 minutes and shown to have $1 \mathrm{GHz}$ frequency fluctations in timescales of a second as displayed in Figure 2b.
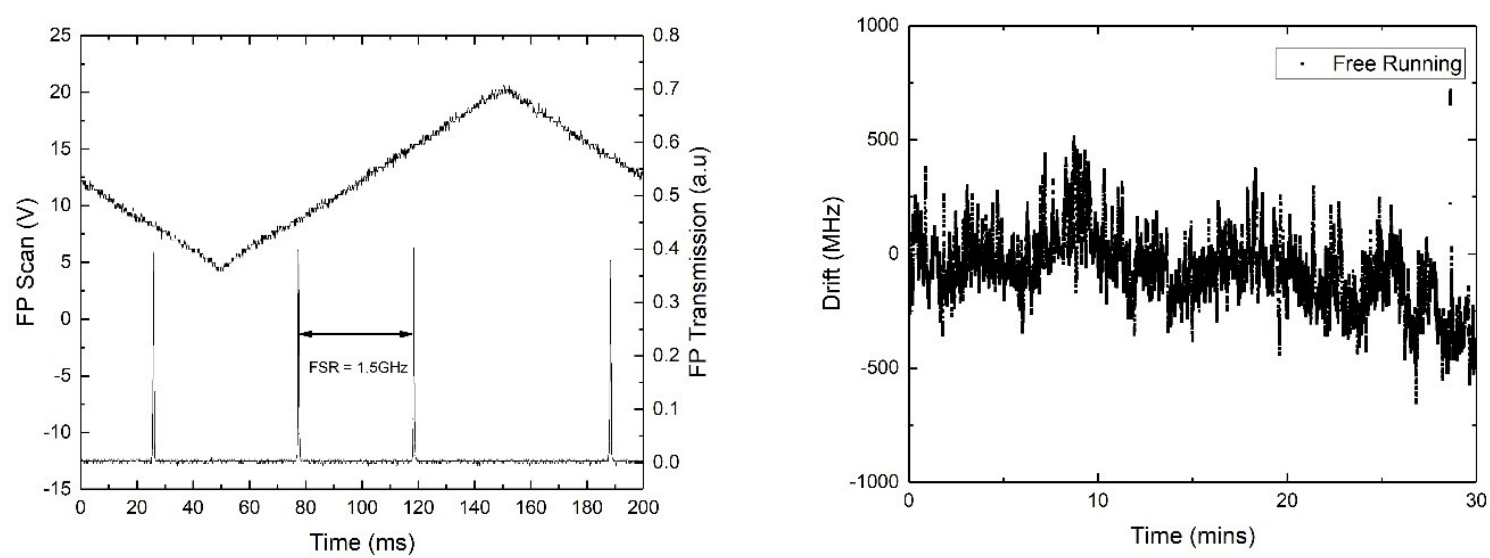

Figure 2. a) Single frequency operation of the SDL. b) Fluctuations in the frequency of the free running laser.

The frequency noise PSD measurement was taken by observing the transmission through a second Fabry-Perot, and monitoring the fluctuations around the halfway point of its resonance. The Fabry-Perot used to observe the fluctuations was a Thorlabs SA200-8B, with a free-spectral range (FSR) of $1.5 \mathrm{GHz}$ and Finesse of $>200$. While the reference cavities were under duress, a Thorlabs SA210-8B, with a FSR of $10 \mathrm{GHz}$ and Finesse of $>150$, was used to give a greater range of measurement that was required due to the vibration, but at the detriment of resolution. The Fabry-Perot was held stable by a Thorlabs MDT693B piezo controller, and the transmission observed by a Thorlabs PDA36A amplified photodetector with a $20 \mathrm{~dB}$ gain setting.

Using a second Fabry-Perot to monitor the fluctuations allow us to examine the system as a whole, including the reference cavity it is locked to. This means that any noise exhibited by the reference cavities under investigation will be apparent, and not just the lasers ability to stabilise to the reference cavity. The noise floor of the detector and contribution from intensity noise is also recorded to determine the influence on the measured linewidths.

The data was captured by a Rohde \& Schwarz RTO1044 oscilloscope, with a resolution of 500 ns and a measurement time of $100 \mathrm{~ms}$. This was then converted into the frequency domain and the frequency noise PSD calculated. An average of ten runs was taken for each frequency noise PSD, averaging after the Fourier transform. The r.m.s. linewidth was calculated 
from this by integrating over a certain period, chosen for this experiment to be between $100 \mathrm{~Hz}$ and $1 \mathrm{MHz}$ unless stated otherwise. The upper frequency constraint was set by the bandwidth of the measurement, with noise quicker than this having negligible effect on the linewidth. In this work, the low frequency was selected to provide a full picture of the contribution from mechanical and acoustic noise sources and relates to a linewidth over a $10 \mathrm{~ms}$ period.
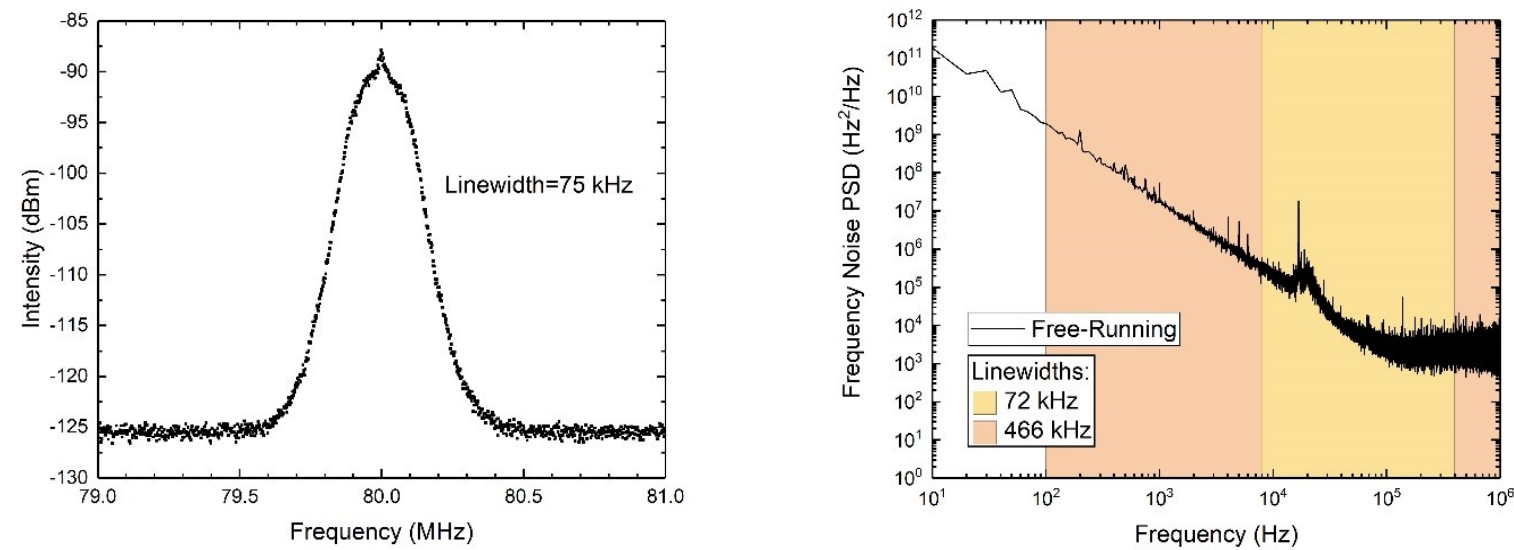

Figure 3. a) Free-running laser linewidth calculated using a self-heterodyne setup. b) Frequency noise PSD of the freerunning laser showing linewidths calculated for different bandwidths.

The linewidth of the free-running laser was calculated from the frequency noise PSD measurement and compared to results acquired using a self-heterodyne setup with a $25 \mathrm{~km}$ delay and an $80 \mathrm{MHz}$ frequency offset by an acousto-optic modulator. The bandwidth of the self-heterodyne measurement is limited; the lower frequency constraint was set by the fibre delay length to be $8 \mathrm{kHz}$, and the upper frequency constraint was set by the bandwidth of the signal analyser, the Rohde \& Schwarz FSV40, at $400 \mathrm{kHz}$ using the sample detection setting. Over the same frequency range the linewidth was calculated from the frequency noise PSD to be $72 \mathrm{kHz}$, comparable to the figure of $75 \mathrm{kHz}$ measured by the self-heterodyne technique. Over a $10 \mathrm{~ms}$ measurement, a linewidth of $466 \mathrm{kHz}$ is calculated. This demonstrates the effect that the bandwidth can have on the linewidth calculation, while also displaying some of the limitations of the self-heterodyne measurement technique.

\section{REFERENCE CAVITY COMPARISON}

\subsection{Active Stabilisation}

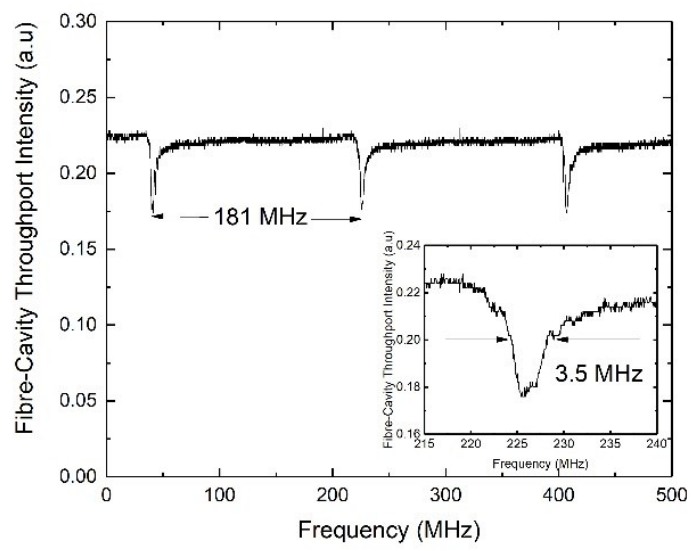

Figure 4. Transmission of the fibre-cavity. 
The transmission of the fibre-cavity is shown in Figure 4. The FSR of the fibre-cavity was calculated to be $181 \mathrm{MHz}$, with a Finesse of 52.

The laser was locked to each reference cavity in turn, the frequency noise PSDs taken, and the linewidths calculated. In Figure 5a, the frequency noise PSD of the system using the fibre loop as a reference cavity is shown, as well as the noise floor of the detector and the noise introduced by fluctuations in the intensity of the laser. It is shown that the frequency noise PSD is limited by detector noise at frequencies above $20 \mathrm{kHz}$, though noise introduced at that magnitude would have little effect on the overall linewidth. It is also shown that the effect of intensity fluctuations on the measurement are negligible.
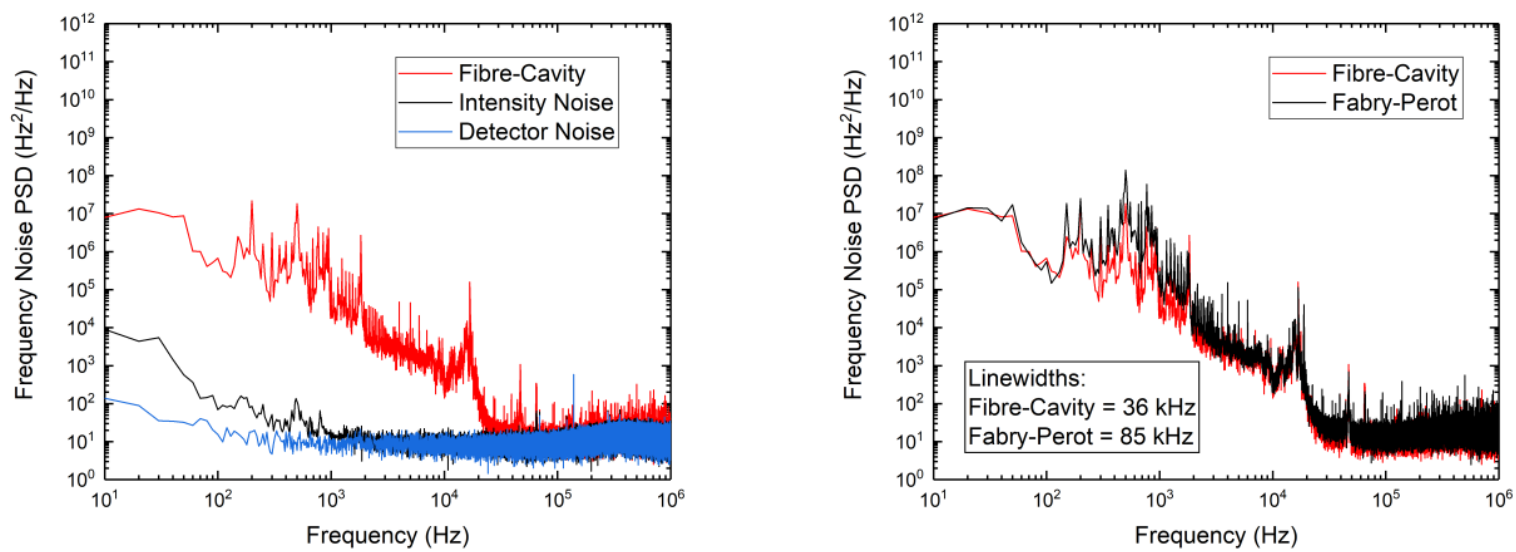

Figure 5. a) Frequency noise PSD of the fibre-cavity showing effect of intensity noise and detector noise. b) Comparison of frequency noise PSDs of reference cavities.

The frequency noise PSD for the two systems is shown to be similar above a frequency of $1 \mathrm{kHz}$, as shown in Figure $5 \mathrm{~b}$, as the lock was too slow above this. The locking bandwidth was limited by the locking electronics used, namely the PI E464 high voltage amplifier. However, at frequencies below this the fibre cavity is shown to operate with lower noise in the system. The linewidth was calculated at $36 \mathrm{kHz}$ while using the fibre cavity as a reference cavity, whereas a linewidth of $85 \mathrm{kHz}$ was calculated while using the Fabry-Perot as a reference cavity.

\subsection{Vibration Testing}

The performance of each reference cavity was tested under vibration by securing it on top of a vibrating platform, the Controlled Vibration ED-3, and suppling this with a pink noise signal generated from Audio Architect software supplied with the platform. An accelerometer was attached to the cavity-baseplate to measure the response of the vibrating platform and confirm its operation. The following frequency noise PSD shows a comparison between the two cavities while pink noise with a gain of $-25 \mathrm{~dB}$ was applied by the software. The fibre-cavity system was calculated to have a linewidth of $876 \mathrm{kHz}$, whereas the Fabry-Perot system was shown to have a worse performance with a linewidth of $1.1 \mathrm{MHz}$. This was repeated for a range of different gain settings and the linewidths resulting from these are shown in Figure $6 \mathrm{~b}$. 

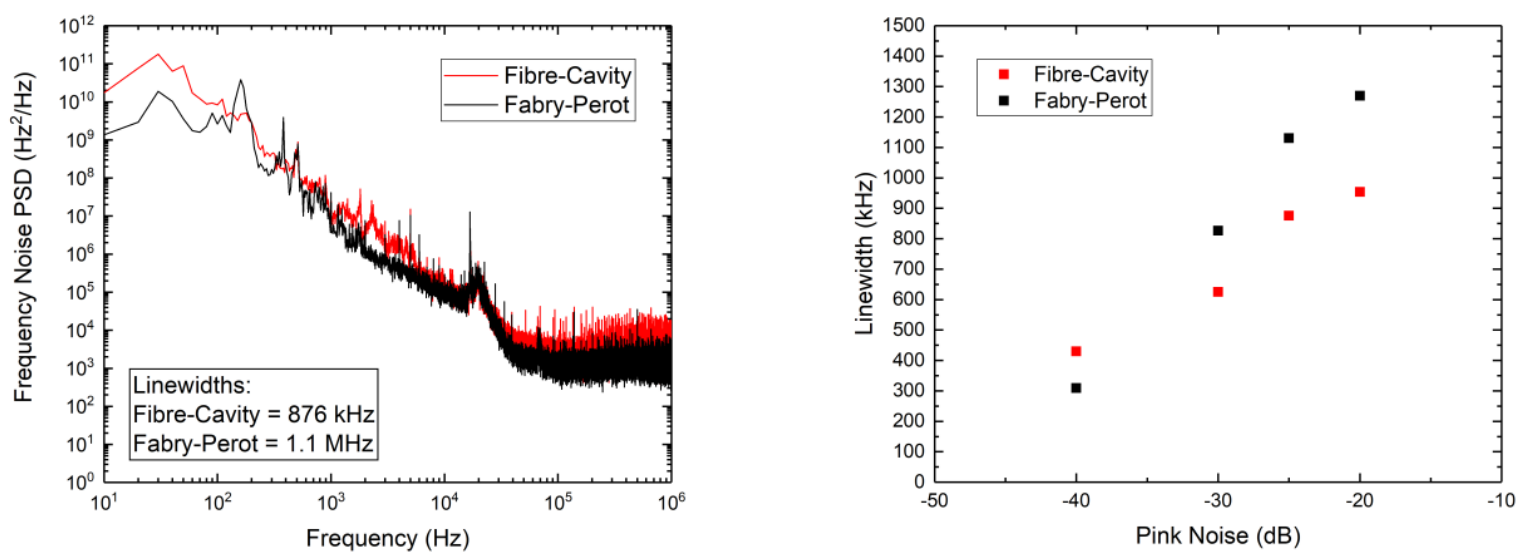

Figure 6. a) Frequency noise PSDs of reference cavities under $-25 \mathrm{~dB}$ pink noise. b) Linewidths calculated from frequency noise PSDs for each reference cavity under a range of vibrations

As can be seen in Figure 6a, and which was consistently witnessed across the range of experimental results, the FabryPerot cavity suffers from noise introduced at $155 \mathrm{~Hz}$. This was examined further by applying a sine wave of $155 \mathrm{~Hz}$ to the vibrating platform. During the experiment, it was evident that the Fabry-Perot was being excited on a resonant frequency, and the resulting frequency noise PSD appears to corroborate this. While the fibre-cavity system actually introduces more noise at $155 \mathrm{~Hz}$, it has superior performance for the majority of the subsequent harmonics, as shown in Figure 7. The resulting linewidths were $1.8 \mathrm{MHz}$ while locked to the fibre-cavity, and $2.3 \mathrm{MHz}$ while locked to the Fabry-Perot.

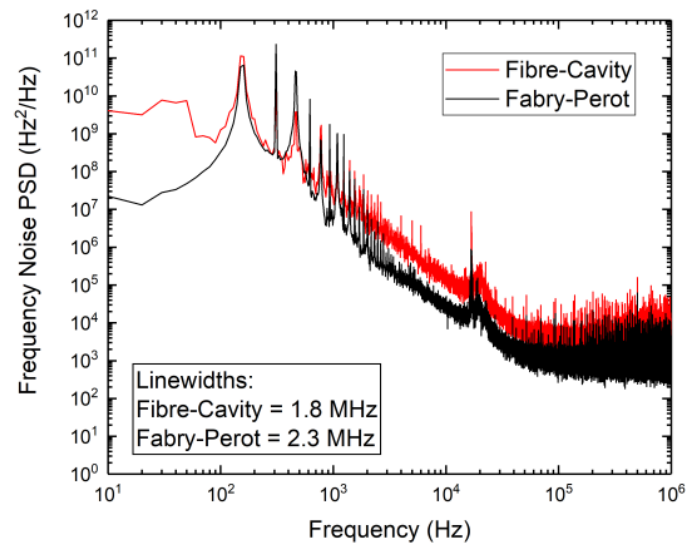

Figure 7. Frequency noise PSD of reference cavities under vibration of a $155 \mathrm{~Hz}$ sine wave.

A similar test was carried out with a sine wave at $100 \mathrm{~Hz}$ applied to the vibrating platform. This time the system locked to the fibre-cavity performed worse, with a linewidth of $2.6 \mathrm{MHz}$, compared to a linewidth of $1.0 \mathrm{MHz}$ resulting from the Fabry-Perot. It should also be noted that the fibre-loop system consistently performed worse at frequencies lower than $100 \mathrm{~Hz}$, and therefore may not be as suitable for experiments requiring a measurement time of over $10 \mathrm{~ms}$ without further stabilisation.

\subsection{Thermal stabilisation}

Each system was capable of remaining locked for over one hour, though environmental control was required by the fibrecavity to attain this length of lock. Insulation was used to enclose the reference cavity and successfully managed to reduce fluctuations in the surrounding temperature and humidity, as well as reducing airflow around the cavity. As a consequence of this, the temperature of the insulated area would steadily increase while the laser was coupled into the fibre-cavity, 
which manifested into a frequency drift due to thermal expansion and an increase in refractive index. The Fabry-Perot however, has been specifically designed to limit these effects, through use of an Invar spacing and expansion compensating mounts, and therefore displayed substantially less drift over extended periods.

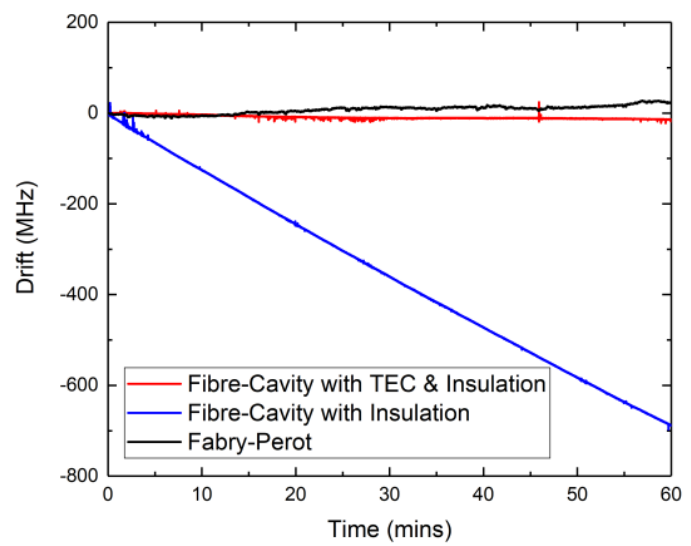

Figure 8. Long-term frequency drift of reference cavities

Through stabilisation of the temperature of the baseplate to which the fibre-cavity is attached we have been able to show that this drift can be controlled. With the simple implementation of a TEC and heatsink, we were able to reduce the drift of the fibre-cavity to $15 \mathrm{MHz}$ over a period of one hour, as shown in Figure 8. This is a clear improvement on the drift of almost $700 \mathrm{MHz}$ witnessed before temperature control was put in place, and even compares favourably to the Fabry-Perot, which experienced a drift of about $30 \mathrm{MHz}$ over the same timeframe.

The thermal control of the waveguide shown here demonstrates the potential to tune the resonance position of the reference cavity. Through direct placement of temperature or stress controlling on the fibre it would be possible to tune this cavity at fast rates.

\section{CONCLUSIONS}

We have developed a novel frequency-stabilisation scheme in the form of a fibre-cavity. With thermal stabilisation in place, it was shown to be an able replacement for the conventional Fabry-Perot reference cavity and in some cases offers superior performance. The fibre-cavity offers a broad transparency with a decoupling between the finesse and alignment. The fibre components are widely available commercially and are lower cost than typical dielectric coated Fabry-Perot cavities. It has shown reduced sensitivity to the majority of vibration tests that were carried out on it, as well as improved stability while in laboratory conditions.

The combination of SDLs and monolithic reference cavities promises to offer narrow-linewidth single-frequency lasers in a low-cost, compact format suitable for a range of applications outwith the confines of the laboratory. The approach is valid at a wide range of wavelengths for which different SDL gain materials are available. Further optimisation of this approach including a higher Q cavity and an exploration of the most appropriate environmental shielding will harvest a truly vibration-insensitive reference cavity. 


\section{REFERENCES}

[1] Holm, M. a., Burns, D., Ferguson, A. I. and Dawson, M. D., “Actively stabilized single-frequency verticalexternal-cavity $\backslash$ AlGaAs laser,” IEEE Photonics Technol. Lett. 11(12), 1551-1553 (1999).

[2] Guina, M., Rantamäki, A. and Härkönen, A., "Optically pumped VECSELs : review of technology and progress," J. Appl. Phys. 50(38), 383001 (2017).

[3] Laurain, A., Mart, C., Hader, J., Moloney, J., Kunert, B. and Stolz, W., “15W Single Frequency Optically Pumped Semiconductor Laser With Sub-MHz Linewidth.,” IEEE Photonics Technol. Lett. 26(2), 131-133 (2013).

[4] Zhang, F., Heinen, B., Wichmann, M., Möller, C., Kunert, B., Rahimi-Iman, A., Stolz, W. and Koch, M., “A 23watt single-frequency vertical-external-cavity surface-emitting laser," Opt. Express 22(11), 12817 (2014).

[5] Pabœuf, D., Schlosser, P. J. and Hastie, J. E., "Frequency stabilization of an ultraviolet semiconductor disk laser.," Opt. Lett. 38(10), 1736-1738 (2013).

[6] Hastie, J. E., Morton, L. G., Kemp, A. J., Dawson, M. D., Krysa, A. B. and Roberts, J. S., “Tunable ultraviolet output from an intracavity frequency-doubled red vertical-external-cavity surface-emitting laser," Appl. Phys. Lett. 89(6), 061114 (2006).

[7] Hopkins, J.-M., Maclean, A. J., Burns, D., Riis, E., Schulz, N., Rattunde, M., Manz, C., Köhler, K. and Wagner,

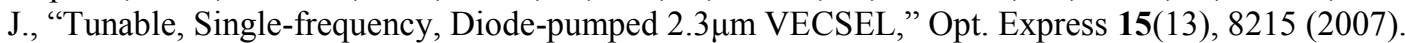

[8] Kaspar, S., Rattunde, M., Topper, T., Rosener, B., Manz, C., Kohler, K. and Wagner, J., "Linewidth narrowing and power scaling of single-frequency 2.X ?? m GaSb-based semiconductor disk lasers," IEEE J. Quantum Electron. 49(3), 314-324 (2013).

[9] Laurain, A., Mart, C., Hader, J., Moloney, J. V, Kunert, B. and Stolz, W., "Optical noise of stabilized highpower single frequency optically pumped semiconductor laser.," Opt. Lett. 39(6), 1573-1576 (2014).

[10] Cheng, Y., Kringlebotn, J. T., Loh, W. H., Laming, R. I. and Payne, D. N., "Stable single-frequency travelingwave fiber loop laser with integral saturable-absorber-based tracking narrow-band filter," Opt. Lett. 20(8), 875877 (1995).

[11] Okoshi, T., Kikuchi, K. and Nakayama, A., "Novel method for high resolution measurement of laser output spectrum," Electron. Lett. 16(16), 630 (1980).

[12] Kemp, A. J., Valentine, G. J., Hopkins, J., Hastie, J. E., Smith, S. A., Calvez, S., Dawson, M. D. and Burns, D., "Thermal Management in Vertical-External-Cavity-Emitting Lasers: Finite-Element Analysis of a Heatspreader Approach,” IEEE J. Quantum Electron. 41(2), 148-155 (2005).

[13] Drever, R. W. P., Hall, J. L., Kowalski, F. V., Hough, J., Ford, G. M., Munley, A. J. and Ward, H., "Laser phase and frequency stabilization using an optical resonator," Appl. Phys. B 31(2), 97-105 (1983).

[14] Black, E. D., “An introduction to Pound-Drever-Hall laser frequency stabilization,” Am. J. Phys. 69(1), 79 (2001). 